\title{
Relaciones personales y politicas de salud en un programa municipal de VIH/Sida de la Provincia de Buenos Aires
}

\author{
AGOSTINA AIXA GAGLIOLO \\ Universidad de Buenos Aires, Buenos Aires, Argentina
}

DOI 10.11606/issn.2316-9133.v28i1p84-107

\begin{abstract}
Resumen El artículo analiza, desde una mirada etnográfica, los modos en que las políticas públicas de VIH/Sida se desarrollan en un municipio del primer cordón poblacional del noroeste del Gran Buenos Aires (Argentina). Atendemos específicamente a lo que los profesionales del Programa Municipal de Prevención y Asistencia denominan "contactos y redes de amistad" y su relación con las respuestas locales a la epidemia. Nos basamos en la reconstrucción y análisis de entrevistas, observaciones, conversaciones e interacciones cotidianas con los profesionales, entre 2016 y 2018. Del análisis se desprende que las relaciones personales son altamente valoradas por estos profesionales en términos de la importancia que tienen para el desarrollo de respuestas político-institucionales al problema del VIH/Sida en el nivel local.
\end{abstract}

palabras clave: políticas de salud; VIH/Sida; Etnografía

Relações pessoais e políticas de saúde em um programa municipal de HIV/Aids na Província de Buenos Aires

Resumo $\mathrm{O}$ artigo analisa, do ponto de vista etnográfico, como as políticas públicas de HIV/Aids são desenvolvidas em um município do primeiro cordão populacional do nordeste da Gran Buenos Aires (Argentina). Atendemos especificamente ao que os profissionais do Programa Municipal de Prevenção e Assistência chamam de "contatos e redes de amizade" e sua relação com as respostas locais à epidemia. Contamos com a reconstrução e análise de entrevistas, observações, conversas e interações cotidianas com profissionais cadastrados entre 2016 e 2018. A partir da análise, percebe-se que as relações pessoais são altamente valorizadas por esses profissionais em termos da importância que têm para o desenvolvimento de respostas político-institucionais ao problema do HIV/Aids em nível local.

palavras-chave: políticas de saúde; HIV/Aids; Etnografia. 


\section{Personal relationships and health policies in a municipal HIV/Aids program}

\section{in Buenos Aires' Province}

abstract This article approaches the ways in which HIV/Aids policies are developed in a municipality located in the northeast of Gran Buenos Aires (Argentina) from an ethnographic scope. It focuses on what the professionals from the Municipal Program for HIV/Aids Assistance call 'contacts and friendship networks', and its connection to local responses given to the epidemic. The argument we propose is based on the reconstruction and analysis of interviews, observations, conversations and quotidian interactions with the professionals registered between 2016 and 2018. From this analysis we convey that such personal relationships are highly valued by these professionals in terms of their significance in the development of political and institutional responses to HIV/Aids at a local level.

keywords: Health policies; HIV/Aids; Ethnography.

\section{Introducción}

El presente artículo busca indagar acerca de las respuestas político-sanitarias vinculadas al VIH/Sida en un municipio de Buenos Aires, atendiendo específicamente a ciertas prácticas que los profesionales del equipo de salud en estudio llaman "contactos y redes de amistad" ${ }^{\prime}$, en las que sostienen y fundamentan discursivamente sus intervenciones. En esta noción incluyen un abanico de relaciones y vínculos con otros profesionales, funcionarios locales e integrantes de organizaciones de personas con VIH/Sida (PVIH).

Se presenta aquí un análisis parcial que integra mi investigación doctoral, consistente en un estudio etnográfico sobre las políticas y tecnologías del VIH/Sida centrado en la experiencia de personas con tratamientos de larga duración. La misma se encuentra en desarrollo desde 2016 en un Programa Municipal de prevención y asistencia del VIH/Sida y adicciones que funciona en un Centro de Atención Primaria de Salud (CAP) del primer cordón del norte-centro del Área Metropolitana de la Provincia de Buenos Aires, Argentina. La primera etapa del trabajo de campo se llevó adelante entre los meses de agosto del 2016 y febrero del 2018, fundamentalmente en el espacio del CAP. La misma incluyó visitas regulares y sostenidas en el tiempo, durante las cuales registré interacciones, conversaciones e intercambios cotidianos en espacios de circulación del público, de atención, de espera y en las oficinas. Asimismo, realicé entrevistas a la totalidad de profesionales de la salud que forman parte del programa (cuatro) y a personas con VIH (PVIH) que se atienden allí o que concurren a actividades organizadas por el programa (trece). Del mismo modo, participé acompañando al equipo de salud en actividades de prevención y promoción de la salud, en

\footnotetext{
${ }^{1}$ Las comillas se utilizan para indicar expresiones/términos/palabras utilizadas por las personas entrevistadas (incluyendo las citas textuales de entrevistas o registros de campo). Los nombres de todos ellos han sido reemplazados por otros ficticios, a fin de preservar el anonimato.
} 
este y en otros CAP del municipio, y en espacios al aire libre. Por último, asistí a reuniones mensuales del "grupo de pares" que una organización de PVIH desarrolla dentro del CAP. Este artículo abreva en los numerosos trabajos que, desde las ciencias sociales, han abordado los procesos de atención y los modos de gobierno de la epidemia en relación al VIH/sida. Los primeros estudios sobre las prácticas y representaciones de profesionales y pacientes, usuarios de drogas, jóvenes y poblaciones de bajos recursos datan de los años 90 y se continúan hasta el presente (BIAGINI, 2000; EPELE, 2003; 2007; GRIMBERG, 1995; 2001; 2002a; 1998; 1997; JONES, 2016; 2017; KORNBLIT, 1997; 1997; MARGULIES, 1996; 1998; PANTELIDES; GOGNA; RAMOS, 2000). También se ha tratado la problemática de vivir con VIH, en diálogo con el bagaje conceptual proveniente de los estudios sobre la vida con enfermedades crónicas, así como los problemas relativos al seguimiento de los tratamientos, la construcción biomédica de la categoría de "adherencia" y los procesos de "expertización" que atraviesan los sujetos (ADASZKO, 2012; BARBER, 2015; GARCIA 2017; GRIMBERG, 2002b; JONES, 2017; MARGULIES, 2010; 2006; PECHENY, 2002; RECODER, 2001, 2011). Lo mismo que los procesos de estigmatización y discriminación vinculados al VIH/Sida en que se ven inmersos algunos grupos sociales (EPELE, 2007; KORNBLIT, 2000; MARGULIES, 2017). Se han abordado asimismo las problemáticas de género y sexualidades en relación al VIH-Sida, analizando también los procesos de embarazo, parto y puerperio de mujeres afectadas (BIAGINI 2008; GARCÍA, 2009; GOGNA et al., 2005; 1997; GRIMBERG, 2000; 2009; PECHENY et al., 2012). Son significativos además los estudios sobre procesos de organización de personas afectadas por el virus desde variados enfoques (BIAGINI, 2009; 1995; GREGORIC, 2008; 2012; 2015; 2017; KORNBLIT, 1999; SANCHEZ, 2013).

Numerosos estudios elaborados desde la Dirección de Sida y ETS del Ministerio de Salud de la Nación, han abordado la caracterización de la población afectada, las dificultades y barreras en el acceso a la atención, los sentidos y significados desarrollados alrededor del VIH y el problema de la "adherencia" a los tratamientos, entre otras cuestiones (BARRÓN LOPEZ, 2008; BIAGINI, 2008; JONES, 2016; 2017; JORRAT et al., 2013; LAPLACETTE et al., 2015; MARGULIES et al., 2010; 2010; MENDES-DIZ, 2008; WELLER et al., 2009). Existen investigaciones de corte similar producidas desde Fundación Húesped ${ }^{2}$ (FUNDACIÓN HUÉSPED, 2011, 2014, 2017; 2011).

El presente escrito recupera asimismo los estudios de las ciencias sociales sobre el Estado y los aportes desde la antropología de las políticas públicas (ABRAMS, 1977; ASAD, 2008; GUPTA, 2015; MITCHELL, 2015), específicamente autores que han marcado la importancia de la perspectiva etnográfica en la crítica de nociones estáticas y reificadas de conceptos como los de política, Estado y gobierno (BALBI, 2008; BALBI; BOIVIN, 2008).

\footnotetext{
2 La "Fundación Huésped" es una organización no gubernamental que desde 1989 trabaja con problemáticas vinculadas al VIH-Sida e ITS. Véase "Misión y Visión” En: Institucional. Fecha de consulta 26/02/2019. $<$ https://www.huesped.org.ar/institucional/mision-y-vision/>.
} 
Se ha señalado también, que la etnografía constituye una herramienta privilegiada para acceder a las prácticas del Estado en la interacción cotidiana de los sujetos con las instituciones y burocracias locales (AUYERO, 2011; GUPTA, 2015; FASSIN, 2015). En esta línea, se ha atendido a aquellas discusiones centradas en las llamadas redes personales en los procesos políticos. A partir del estudio de las prácticas de integrantes de movimientos sociales, algunas autoras han cuestionado la prevalencia de una mirada instrumental sobre el accionar de los sujetos que hacen políticas, particularmente en trabajos que han analizado el fenómeno del clientelismo politico (AUYERO, 2001, 2002; en MANZANO, 2013). Señalando la falsa dicotomía que otorga centralidad al intercambio instrumental como verdadero fundamento de las prácticas - transformando las dimensiones morales y afectivas de estos lazos en simples creencias, representaciones o ideologías - (AUYERO, 1997; 2001; 2002; 2007; GRIMSON, 2003 en VOMMARO; QUIRÓS, 2011), autoras como Julieta Quirós (2008) abordan la dimensión afectiva del hacer cotidiano, como elemento fundamental en el origen y la continuidad de la participación política.

Nos proponemos aportar al conjunto de investigaciones que se han centrado en las prácticas institucionales de los sujetos que implementan, crean y modelan las políticas públicas, insertos en el proceso que constituye la trama misma de relaciones que llamamos Estado (CANELO, 2008; FASSIN, 2015; GAZTAÑAGA, 2008; QUIRÓS, 2014; SHORE, 2010). Recuperamos para ello mis observaciones acerca de las prácticas cotidianas y los relatos de profesionales que componen el "Programa municipal de asistencia y prevención del VIH/Sida y la drogodependencia” bajo estudio. Analizamos allí cómo se configura el entramado de relaciones interpersonales que estos profesionales - y agentes estatales establecen, y cómo su accionar ha dado forma a las respuestas político-sanitarias locales a la epidemia.

El presente artículo se organiza en tres apartados. El primero de ellos aborda los relatos que los profesionales realizan sobre los inicios del programa y las relaciones profesionales y políticas que dieron origen al mismo. El segundo, toma la cuestión de las relaciones con los organismos de financiamiento y cooperación internacional, el Estado nacional y las organizaciones de personas con VIH y los modos en que las mismas transformaron las dinámicas del Programa Municipal a lo largo de su desarrollo. El tercero se centra en las prácticas de atención cotidianas; en cómo las "amistades" y los "favores" dan forma a una manera particular de ejercer y concebir la atención a la salud. Por último, la conclusión se aproxima a la mirada de estos profesionales sobre el Estado, comprendido como un entramado de relaciones en el cual se insertan activamente.

\section{El programa municipal}

El "Programa municipal de prevención y asistencia del Sida y la drogodependencia", como lee la ordenanza municipal que dicta su creación, funciona en un Centro de Atención Primaria de Salud (CAP) de dependencia municipal. Se trata de un municipio ubicado en el primer cordón poblacional del norte-centro (o noroeste, dependiendo de la fuente) del Gran 
Buenos Aires (Área Metropolitana de Buenos Aires) y tiene unos 340.000 habitantes distribuidos en $45 \mathrm{~km}^{2}$ de superficie. Se trata de un municipio fundamentalmente urbano, parte de una subregión de perfil industrial cuyos indicadores de Producto Bruto Geográfico (PBG) han sido calificados como altos y sus niveles de carencia por Necesidades Básicas Insatisfechas (NBI) se encuentran entre los más bajos del conurbano bonaerense, haciendo del mismo un municipio con un nivel de recursos elevado (COUTO, 2018).

El programa fue creado en 1992, muy tempranamente en la historia de las respuestas político-sanitarias al VIH/Sida en la Argentina, antes incluso de que se conformara el Programa Nacional de Lucha contra el SIDA y ETS ${ }^{3}$. Desde que el programa se constituyó como tal, se encuentra emplazado en el CAP más grande, importante y mejor aprovisionado del Municipio ${ }^{4}$, cuya ubicación es estratégica dentro de la geografía municipal: se encuentra a sólo una cuadra del edificio de la Municipalidad - el centro político del municipio - en la localidad "cabecera" (central) del partido. Parte del espacio físico del CAP está destinado a oficinas que albergan autoridades municipales - secretaria y subsecretarias de Salud y Desarrollo humano y directores de programas pertenecientes a esas secretarías (Dirección de Epidemiología, Dirección de Atención Médica, Dirección de Vectores, entre otros), y sus respectivos empleados administrativos. Allí se centraliza una gran parte de los trámites administrativos de todo el personal de salud municipal. Dadas estas coordenadas espaciales, las personas que trabajan en el CAP interactúan cotidianamente con autoridades y funcionarios, produciéndose una fluida circulación de información sobre cambios en cargos políticos, despidos, reemplazos, dificultades en la provisión de insumos, etc. Del mismo modo, es frecuente el contacto con agentes de otras dependencias municipales (otros CAP) o incluso del propio "edificio", nombre que le dan a la Municipalidad. La circulación de rumores, comentarios y chismes sobre el municipio ocupa un lugar importante en las conversaciones cotidianas de sus trabajadores.

El programa de VIH, según señalan sus creadores, estuvo originalmente constituido por alrededor de catorce profesionales provenientes de diferentes áreas del Municipio, de los cuales sólo tres se encuentran aún trabajando allí. Actualmente está integrado por Oscar (psicólogo), Matilde (psicóloga), Merlina (médica infectóloga) y Camila (trabajadora social que se incorporó a finales del año 2015). Una particularidad de este Programa es que además de las tareas vinculadas a la gestión estatal en salud, también brinda atención médica, psicoterapéutica, orientación en demandas sociales y dispensa de medicación a personas con

\footnotetext{
${ }^{3}$ En 1990 se promulgó la Ley Nacional de Sida (Ley Número 24789, 1990) y dos años después se promulgó la ordenanza municipal que dictó la creación de este Programa Municipal. En 1994 se crea el Programa Nacional de Lucha contra el SIDA y Enfermedades de Transmisión Sexual (ETS), que comenzaría a realizar la distribución de los medicamentos disponibles para el tratamiento. Algunos integrantes del Programa Municipal remarcan que se trató del "primer programa de atención primaria en VIH". Hemos relevado la existencia de un programa municipal lindante también creado en 1992. El mismo no funciona como espacio de atención.

${ }^{4}$ La oferta de atención del CAP incluye: estudios de imagen, laboratorio, clínica médica, pediatría, neonatología, urología, neurología, cardiología, cardiología infantil, psiquiatría, psicología para adolescentes, adultos y niños, kinesiología, reumatología, fonoaudiología, neumonología de adultos y niños, servicio social.
} 
VIH. Este doble carácter es indesligable de sus orígenes: quienes conformaron el programa ya se encontraban trabajando como profesionales de la salud dentro del CAP; la institucionalización del programa como tal les permitió formalizar una actividad que venían realizando "a pulmón" desde mediados de los años '80, y comenzar a desarrollarse dentro de la gestión.

En el marco del trabajo de campo, los profesionales del equipo de salud refirieron y reiteraron tanto en las entrevistas como en el curso de interacciones cotidianas, que su tarea se sostiene en "redes" de relaciones tejidas por ellos. En la cotidianeidad observé cómo parte de su tarea consiste precisamente en sostener y construir esos vínculos. Se trata de relaciones diversas: de amistad, de cortesía, de afinidad ideológica, de compañerismo, de solidaridad, o de mutua conveniencia. Unas involucran algún grado de afecto, otras el compromiso militante o las convicciones ideológicas, otras son parte del sostén cotidiano de los servicios. Estas relaciones se establecen con personas ubicadas en diferentes jerarquías: sujetos que poseen cargos políticos relevantes, agentes del estado provincial y nacional, compañeros del CAP, otros profesionales de la salud, integrantes de organizaciones de PVIH e integrantes de asociaciones de autoayuda como Narcóticos Anónimos. Todas ellas podrían ser caracterizadas como relaciones personales que, aunque no están estrictamente formalizadas en el entramado institucional en el que los actores se encuentran insertos, se entrelazan con éste de forma permanente y cotidiana.

Distintos autores han recuperado la relevancia de aquello que denominan relaciones informales o redes informales en la operatoria de los servicios de salud (ROVERE, 1999), llamando la atención sobre el problema de los mitos racionalistas sobre la creación de políticas de salud en el seno del Estado (SPINELLI, 2017). Algunas lecturas señalan que éstas deben ser aprovechadas en el mejoramiento de la gestión y administración de la salud pública (MARQUÉS SÁNCHEZ et al., 2012). Otras comprenden a estas relaciones como producto de la solidaridad entre trabajadores de la salud que buscan hacer frente a los contextos de precariedad institucional en los que se encuentran insertos, pero que a largo plazo contribuyen al deterioro estructural del sistema público de salud, posibilitando su conversión en instrumentos de politicas clientelares (CROJETHOVIC, 2012). García y otras han señalado la relevancia de las relaciones entre profesionales "viejos conocidos" al momento de derivar/recibir un paciente en un contexto hospitalario y cómo impactan en el recorrido institucional de las personas que acuden en búsqueda de atención (GARCÍA et al., 2017). En este artículo proponemos abordar estas relaciones no formalizadas institucionalmente que se establecen al interior del sistema de salud en diálogo con el campo de estudios sobre antropología del Estado y las políticas, abriendo nuevas posibilidades para analizar este fenómeno. En este marco, algunas autoras han mostrado que las relaciones personales son una pieza clave en la comprensión de las políticas en múltiples espacios (CANELO, 2008), e incluso que es la articulación de las mismas con el mundo institucional la que permite a ciertos sujetos producir políticas (GAZTAÑAGA, 2008). Así, ahondar en el accionar cotidiano de los agentes estatales, en los modos en que éstos simultáneamente 
implementan y producen políticas públicas, a la vez constreñidos por el marco institucional y dotados de capacidad creativa, nos abre -como plantea Didier Fassin (2015) - una rendija por la cual observar los modos en que el Estado se constituye como tal.

En nuestro caso, describimos y analizamos cómo las formas en que se desenvuelven las relaciones personales - aquí denominadas "contactos y redes de amistad" - modelan las respuestas locales al VIH/Sida. En este objetivo resulta central la perspectiva etnográfica, que permite atender a las lógicas que sostienen las prácticas que dan forma y sentido al Estado (BALBI 2008b).

\section{Relaciones personales en el origen y desarrollo del programa municipal}

Según sus creadores el Programa nació por la necesidad de brindar respuesta a la demanda de atención existente en el municipio, en un momento en el cual el VIH aún era una enfermedad con altísima letalidad y con pocas posibilidades de respuesta biomédica eficaz (MARGULIES, 2010) ${ }^{5}$. Sus fundadores eran psicólogos, trabajadoras sociales y médicas de varias especialidades, que en algún momento se encontraron durante la práctica cotidiana y forjaron vínculos a partir de los desafíos que les colocaba la epidemia. En palabras de Oscar, fue la aparición de "pacientes con características poco habituales, casos raros, gente con kaposi, micosis, cosas que nadie sabía por qué eran” lo que los llevó a “comunicarse en los pasillos" y empezar a elaborar estrategias colectivas que permitieran hacer frente a la situación. Estos lazos comenzaron a tomar forma a mediados de 1980 y comienzos de 1990 entre profesionales dentro del municipio, muchos de los cuales eran trabajadores de salud del mismo CAP. También ocurrieron movimientos similares a escala de la provincia de Buenos Aires (y en el nivel nacional), donde éstos y otros profesionales comenzaban a reunirse para conformar aquello que luego sería la Sociedad Argentina Interdisciplinaria de Sida (SAISIDA) ${ }^{6}$.

Retomaré en detalle los relatos de Matilde y Merlina para analizar el origen del programa, por tratarse de quienes más historias han compartido conmigo en ese sentido, y por ser ambas integrantes que se destacan dentro de este espacio. Recupero también algunos aportes de Oscar, quien forma parte del programa desde sus inicios.

Matilde es una mujer de unos 55 años, morocha, de pelo canoso, es simpática pero también muy incisiva en sus comentarios y apreciaciones. Se reconoce a sí misma como

\footnotetext{
${ }^{5}$ Margulies analiza los modos en que, con el desarrollo de los primeros casos que involucraban a personas afectadas por el VIH/Sida en Argentina, un conjunto de médicos se organiza para brindar respuestas a los mismos. La autora enfatiza en las dificultades que implicaba la atención en momentos donde aún no había tratamientos antirretrovirales efectivos, y en los que el Sida era considerada una enfermedad terminal con alta tasa de mortalidad.

${ }^{6}$ La Sociedad Argentina Interdisciplinaria de Sida (SAISIDA) es una sociedad sin fines de lucro que tiene como característica distintiva la asociación de profesionales de la salud de distintas disciplinas (médicos, enfermeros, consejeros, psicólogos, sociólogos, antropólogos, etc.), miembros de organizaciones no gubernamentales y organizaciones de la sociedad civil y personas viviendo con VIH/SIDA (PVVS) comprometidos con el trabajo en VIH/SIDA. Véase SAISIDA. "s.f.". ¿Qué es Saisida? Fecha de actualización s.f. Fecha de consulta 4/6/2018 11:15hs. $<$ www.saisida.org $>$.
} 
"peronista de toda la vida", y considera que la política es una parte muy importante de su vida cotidiana. Matilde me integró a su red de relaciones en los mismos términos que la he visto interactuar con otros; siempre se mostró amable, generosa y dispuesta a conversar conmigo. Entre sus tareas al interior del programa, Matilde dice disfrutar más de aquellas que involucran la gestión de recursos o proyectos, y menos de la atención clínica. Es psicóloga del Programa Municipal de VIH desde el año 1993 y frente a mi pregunta sobre la formación del programa, me dijo:

Esto surgió a partir de la inquietud de Merlina que laburaba acá [...] como médica en el Mercado ${ }^{7}$ y [...] en los ‘ 90 empieza a ver que empiezan a venir un montón de personas que vivían con VIH. Quien en ese momento estaba en el co-gobierno de ese Mercado era quien fue secretario de salud de acá del municipio durante mucho tiempo y secretario de desarrollo [social] [...] Supongo que, a partir de la influencia de Merlina, no sabemos por qué se sensibilizó con esta temática [...] y decide convocar a un montón de profesionales a trabajar en un programa de prevención y asistencia del Sida y la drogodependencia (Entrevista Matilde $23 / 11 / 2016)$

En este fragmento Matilde sintetiza nuestras variadas conversaciones sobre el nacimiento del programa y la "influencia" inicial de Merlina. Su relato invita a considerar que es Merlina quien, a través del vínculo personal con el secretario de salud, realiza el gesto fundacional del programa impulsada por la necesidad de brindar respuestas a la demanda de la población.

Merlina tiene más de 60 años, es una mujer de baja estatura que se refiere a sí misma como "gordita". Tiene un gesto amoroso y amable, se comporta de modo maternal casi con todas las personas que la rodean; actitud que también adoptó conmigo. Es oriunda de Santiago del Estero, pero vive en Buenos Aires desde el inicio de su carrera universitaria, cuando su familia se relocalizó para que ella y su hermana pudieran estudiar. En numerosas ocasiones la escuché hablar con cariño de su padre, "un ferroviario medio socialista, medio peronista" que la impulsó a tener "compromiso social" en "contextos de pobreza". Frecuentemente se jacta de haber tenido una "formación sanitarista" que le enseñó a "priorizar la atención primaria de la salud, a tener una visión más general e involucrar toda la parte social y económica del paciente" (Registro de campo 71, 18/09/18), mirada que señala con preocupación- ya no existe entre los médicos jóvenes. También es habitual que recuerde cuando "era jovencita" y realizó el Curso Superior de Infectología en el Hospital

\footnotetext{
${ }^{7}$ El "mercado" es un espacio donde se realiza la comercialización mayorista de frutas y verduras. Allí trabajan y circulan cotidianamente una cantidad considerable de personas y es significativo en la provisión de estos alimentos a nivel municipal.
} 
Muñiz $^{8}$. Trabaja en el municipio desde mediados de la década de 1980 (cuando realizaba guardias en el mismo CAP). En el año 1990, a raíz de una epidemia de cólera, la convocaron a incorporarse al trabajo en el mercado de frutas y verduras municipal. Dice sobre la creación del programa:

En el año '92 con tres médicas, no, éramos cinco, perdón, una dermatóloga, una inmunóloga, la neumonóloga y yo, decidimos armar un programa con los psicólogos, el primer programa de atención primaria en VIH. Teníamos un buen equipo [...] con los psicólogos y trabajadores sociales. Por eso lo decidimos armar. (Entrevista Merlina 24/10/2016).

Oscar, el psicólogo del equipo, tiene más de 60 años, es delgado, de baja estatura y ojos claros. Frecuentemente su condición de "rubio" (de tez y cabello claro) es motivo de bromas por parte de sus compañeras. Siempre afable y comunicativo, aprovecha cada ocasión para mostrar fotos de su nieta de dos años. Oscar se dedica fundamentalmente a la atención clínica, y sus pacientes varían entre jóvenes y adolescentes de escuelas de oficio, personas con consumo problemático de sustancias y personas con VIH. Comenzó a trabajar en el municipio en 1985 y sus tareas no variaron desde entonces, incluso después de la institucionalización del programa, al que se dedicó exclusivamente. Cuando habla de la creación del mismo, Oscar siempre refiere el trabajo conjunto de profesionales y sus preocupaciones frente a la epidemia. Expresa la formalización del programa como un logro: "el armado del programa y la sanción por decreto municipal es el reconocimiento de una realidad que nos trajo bastante trabajo" (Entrevista Oscar 24/02/2017).

En los relatos de todos los profesionales se mencionan circunstancias significativas. En un contexto de escasas respuestas técnicas y alta incertidumbre en la atención de personas afectadas por el VIH/Sida, se narra la construcción de relaciones de solidaridad y de afinidad profesional que calificaron como "redes de amistad". Esta construcción puede inscribirse en el proceso de configuración de lo que se ha denominado "los médicos del Sida" (MARGULIES, 2014) refiriendo a aquel conjunto de profesionales que respondieron a los primeros casos de Sida en la Argentina y se comprometieron en la búsqueda de respuestas frente a las necesidades de sus pacientes, enfrentando los procesos de estigmatización y discriminación y resignificando su campo de ejercicio tanto al nivel técnico como moral.

En los relatos sobre el programa se destaca asimismo la importancia de los "contactos" de Merlina con autoridades y funcionarios municipales dentro de su territorio, tales que le permitieron "influir" en las decisiones político-sanitarias municipales. Esta última caracterización es más propia de Matilde que de Merlina, ya que la segunda - al igual

\footnotetext{
${ }^{8}$ El Hospital de Infecciosas "Dr. Francisco Javier Muñiz" es un hospital monovalente de la Ciudad Autónoma de Buenos Aires. Se especializa en enfermedades infecciosas y tuvo un rol destacado en la historia del VIH en la Argentina, por encontrarse entre los primeros establecimientos que organizaron respuestas médicas en el país. Asimismo cuenta con una de las especializaciones en Infectología más prestigiosas del territorio nacional.
} 
que Oscar - frecuentemente coloca el énfasis en las otras relaciones personales, las de "amistad" con profesionales que buscan una solución colectiva al problema del Sida. Pero sea que el énfasis esté colocado en la figura de Merlina, o en el conjunto de profesionales, en los relatos acerca del origen del Programa Municipal, las relaciones personales entran en escena como aquel elemento que habilita la puesta en marcha de una respuesta político-institucional al problema del VIH/Sida en el nivel local, volviéndose indisociables de la propia gestión estatal.

El análisis de estas relaciones personales nos muestra estrategias propias de Merlina, Oscar y Matilde (y del resto de los profesionales), pero también nos permite abrir -como anticipamos- una rendija por la cual espiar el funcionamiento del Estado (Fassin, 2015).

Sobre el desarrollo del Programa Municipal, Matilde destaca:

Nosotros crecimos con una gestión que fue el peronismo [...] [con la que] logramos bastante independencia y autonomía [...] si bien había un montón de cosas en las que no acordábamos y notábamos que quizás teníamos que recibir más apoyo, ya en los ultimos años decíamos 'ay, ya con que no nos jodan y nos dejen hacer es suficiente' y aprovechábamos por ahí [...][llegando] hasta tener contacto directo con nación sin pasar por la región ni por la provincia. Por los contactos y por las redes de amistad básicamente que tiene Merlina éramos bien recibidos en todos lados y teníamos acceso (Entrevista Matilde 23/11/2016) ${ }^{9}$.

Aquí "independencia y autonomía" refieren a la posibilidad de interactuar con actores de diferentes instancias gubernamentales, pero también de diseñar las políticas municipales desde el propio programa, sin depender de la aprobación de las autoridades locales en cada paso. Por ejemplo, ejecutar proyectos con organizaciones de $\mathrm{PVIH}^{10} \mathrm{u}$ organizar actividades de prevención en espacios municipales. Autores como Fassin (FASSIN, 2015) señalan la importancia de comprender que los agentes insertos en el Estado con frecuencia se salen de los límites de sus competencias, esto es, no solamente tienen libertad para interpretar e implementar las políticas públicas sino que cuentan con una autonomía relativa que les permite crear nuevas modalidades políticas estatales. Podríamos señalar que estos sujetos no solamente cuentan con autonomía relativa, sino que hacen uso de la misma a sabiendas del impacto que ello tiene en las vidas de las poblaciones que son su objeto de intervención. En el caso del Programa Municipal, tal autonomía es comprendida

\footnotetext{
${ }^{9}$ El resaltado es nuestro.

${ }^{10}$ Los proyectos financiados por entidades internacionales fueron disminuyendo antes del 2015, en parte debido a que las mismas agencias comenzaron a financiar otro tipo de actividades y en parte por la disminución del impacto de las mismas dentro del VIH en Argentina, que sobrevino a la estabilización de la epidemia y la recuperación parcial del sistema de salud.
} 
y definida por los agentes en relación con las condiciones que colocan las autoridades del municipio, y revalorizada ante la posibilidad de perderla.

A finales del 2015, luego de las elecciones a nivel nacional y local, un nuevo intendente pasó a estar a cargo del municipio, con un alineamiento político-partidario diferente al anterior ${ }^{11}$. Matilde me señaló reiteradamente que esto impactó en el municipio y en el propio programa, haciéndome notar en cada situación cotidiana que lo ameritaba cómo se habían limitado las actividades por una serie de nuevos requisitos burocráticoadministrativos, como la obligatoriedad de solicitar por escrito los insumos y/o espacios para realizar actividades.

Con los cambios políticos a nivel nacional, también se modificaron algunos "contactos" de los profesionales del programa. Mientras unos dejaron de estar disponibles, otros -como aquellos que se establecen con agentes estatales de diferentes jerarquías (profesionales del Programa de VIH/Sida del Ministerio de Salud de la Nación, por ejemplo) - pudieron sostenerse con ciertas limitaciones. Incluso, algún “amigo" pasó a ocupar un cargo importante dentro de la estructura estatal, trayendo consigo nuevas posibilidades. Así, las relaciones que son foco de este análisis se muestran dinámicas: mientras ciertos lazos permanecen o desaparecen; otros se crean, reformulan o replantean, sobre todo atendiendo al sostenimiento de las tareas cotidianas.

\section{Las redes de personas con VIH y el financiamiento de proyectos}

Entre aquellos actores que conforman los "contactos y redes de amistad" que colaboraron a la creación y el mantenimiento del Programa Municipal, los profesionales incluyen también a personas con VIH: pacientes y/o miembros de organizaciones no gubernamentales.

Es de importancia señalar que una de las particularidades de la epidemia del VIH/Sida a nivel mundial es la relevancia que las personas afectadas han tenido en la promoción y desarrollo de políticas, exigiendo la provisión de medicamentos antirretrovirales, el acceso a diagnóstico y atención adecuados, etc. Esto puede relevarse en una amplia producción de las ciencias sociales (cf. BIEHL, 2011; FASSIN, 2007; GREGORIC, 2012; 2017; LOWY, 2000; NGUYEN, 2004; VALLE, 2002; 2015). El caso de este Programa Municipal no es la excepción, ya que el mismo fue - y continúa siendo - un espacio de concurrencia y demanda de una de las organizaciones de personas con VIH más importantes de la provincia de Buenos Aires, y la sede de múltiples proyectos conjuntos con esta organización.

Hacia mediados de la década de 1990, el Banco Mundial, el Fondo Global ${ }^{12}$,

\footnotetext{
${ }^{11}$ Durante más de 20 años el Municipio se encontró bajo la dirección del Peronismo, con un mismo intendente a cargo. En el 2015 el Frente Para la Victoria perdió las elecciones frente al mismo partido que resultó vencedor en las elecciones a nivel nacional, el PRO "Propuesta Republicana". Esto resulto en un cambio total de las autoridades intermedias a nivel municipal (secretarios, subsecretarios, directores de programas, directores de CAP).

${ }^{12}$ El Fondo Global es una asociación público-privada mundial dedicada a la recolección y distribución de recursos para prevenir y tratar el VIH y el SIDA, la tuberculosis y la malaria. Véase "Mecanismo Coordinador País. Fondo global de lucha contra el Sida, la Tuberculosis y Malaria.” en: OPS/OMS Argentina. Fecha de actualización:
} 
ONUSIDA $^{13}$ y otros organismos financieros y de cooperación internacional aportaron al desarrollo de programas y proyectos destinados a abordar la cuestión del VIH/Sida en la Argentina. Uno de los más importantes fue el proyecto LUSIDA ${ }^{14}$ bajo el cual se desarrollaron numerosos proyectos locales con participación de la "sociedad civil". La crisis económica que afectaba al país, y las políticas neoliberales, proporcionaban en ese momento el escenario ideal para la intervención este tipo de organismos, que funcionaron en asociación con el Estado nacional, cuestión analizada por autoras como García en Argentina (GARCÍA, 2017) y Sullivan en Tanzania (SULLIVAN, 2012).

Es destacable que los responsables de la gestión estatal de estos proyectos en Argentina (elaboración, establecimiento de prioridades, capacitación, seguimiento y monitoreo) formaban parte también de las "redes de amistad" de Merlina y Matilde. Se trata de médicos infectólogos, muchos compañeros de facultad o residencia de Merlina, y otros profesionales con funciones al nivel del Ministerio de Salud de la Nación o la Ciudad Autónoma de Buenos Aires y/o dentro de las propias agencias internacionales. Fue a través de uno de los "amigos" de Merlina, director del servicio de infectología de un importante hospital nacional, que el Programa Municipal fue convocado hacia 1997 para elaborar proyectos que serían financiados por el proyecto-marco LUSIDA. Este "amigo" había sido compañero de Merlina en el Curso Superior de Infectología en el Hospital Muñiz varios años atrás: cuando fue convocado para elaborar el primer proyecto, se contactó con tres CAP cercanos, incluyendo al Programa Municipal. En la dirección de estos proyectos a nivel nacional se encontraba otro compañero de Merlina. Así, estas relaciones abrieron la posibilidad de participar en proyectos con fondos internacionales, lo cual, al mismo tiempo posibilitó el conocimiento de nuevos actores que también se tornarían significativos en las trayectorias de los profesionales y del propio programa. Luego del primer proyecto LUSIDA,

18/03/2014. Fecha de consulta: 15/07/2018. <https://www.paho.org/arg/index.php?option=com _content\&view=article\&id=555:mecanismo-coordinador-pais-fondo-global-lucha-contra-sida-tuberculosismalaria\&Itemid $=410>$.

${ }^{13}$ El Programa Conjunto de las Naciones Unidas sobre el VIH/Sida: "lidera el esfuerzo mundial por poner fin a la epidemia de sida como amenaza para la salud pública para 2030 como parte de los Objetivos de Desarrollo Sostenible”. Véase “QQuiénes somos?”. En: About UNAIDS. Fecha de consulta 15/07/2018. $<$ http://www.unaids.org/es/whoweare/about>. Para un listado de las políticas promovidas por este organismo dependiente de la Organización de las Naciones Unidas véase también "Recursos". En Resources UNAIDS. Fecha de consulta 18/04/2018 <http://www.unaids.org/es/resources>.

${ }^{14}$ El Proyecto de Lucha contra el SIDA y Enfermedades de Transmisión Sexual fue co-financiado entre el Banco Mundial y el Ministerio de Salud de la Nación. Funcionó entre 1997 y 2000, sus actividades luego se integraron al Programa Nacional de Sida y ETS. Para acceder al balance sobre las actividades comprendidas por este proyecto véase Ministerio de Economía. 2001. Evaluación del Programa Nacional de Lucha contra el SIDA y Enfermedades de Transmisión Sexual. Dirección de Gastos Sociales Consolidados. Buenos Aires: Ministerio de Economía. Fecha de consulta 15/07/2018. <https://www.economia.gob.ar/peconomica/basehome/sida.pdf>. 
continuaron desarrollando otros proyectos con organismos como $\mathrm{GTZ}^{15}$ en 1998 y el PNUD $^{16}$ en el $2000 / 2001$.

Un requisito fundamental que las agencias de cooperación internacional colocaban para la aprobación de las propuestas locales era la vinculación del Estado con "organizaciones de la sociedad civil”. En este contexto, el Programa Municipal comenzó a establecer relaciones con una de las redes de PVIH de la provincia de Buenos Aires, cuyos referentes eran sus pacientes. Con ellos continuaron desarrollando proyectos por casi una década y fue en ese proceso que constituyeron relaciones personales de "amistad", particularmente estrechas, que implican afectos y cuidados sostenidos hasta la fecha ${ }^{17}$. Este fenómeno muestra cómo motivaciones prácticas resultantes de las exigencias de las agencias internacionales y la dimensión afectiva de los lazos emergentes se encuentran enmarañadas en la construcción y el sostenimiento del Programa.

El equipo de profesionales recupera el valor de estos proyectos conjuntos porque los mismos dotaron al programa de prestigio dentro del municipio, les permitieron expandir su territorio de influencia hacia otras instituciones (como escuelas y diferentes espacios culturales), y también acceder a procesos de formación profesional. Matilde señala durante su entrevista:

[...] nosotros éramos contraparte [desde el] Estado, ellos nos daban capacitación técnica, insumos, nos apoyaban desde todos esos lugares y nosotros éramos los que teníamos el laburo ${ }^{18}$. Pero la condición era que nosotros nos asociáramos como contraparte con la sociedad civil, y ahí fue cuando hacemos estos lazos con algunos de nuestros pacientes que eran de la [organización de PVIH] [...] y nos empezaron a capacitar en gestión de proyectos sociales y la verdad que me encantó, dije 'qué bueno que está esto' y daba como otro vuelo y era estar en territorio, con la gente, armando otras cosas, era muy piola ${ }^{19}$. (Entrevista Matilde 23/11/2016).

Al mismo tiempo, como señaló Matilde en numerosas conversaciones, el trabajo con organizaciones de PVIH les "abrió la cabeza" y les permitió “repensar el trabajo” dentro del

\footnotetext{
${ }^{15}$ Grupo de cooperación técnico argentino-alemán - Deutsche Gesellschaft für Technische Zusammenarbeit (GTZ o GIZ). Véase: «About GIZ». Fecha de consulta: 15/07/2018. <www.giz.de/en/html/about_giz.html>.

${ }^{16}$ Programa de Naciones Unidas para el Desarrollo. Véase: «El PNUD en Argentina». Fecha de consulta: 15/07/2018. $<$ http://www.ar.undp.org/content/argentina/es/home.html>.

${ }^{17}$ En numerosas ocasiones observé a las profesionales del programa interactuar con referentes de esta organización, allí se hizo evidente que se trata lazos estrechos que dan cuenta de vínculos prolongados en el tiempo y cargados de afectividad. Es habitual la presencia de estos sujetos en espacios reservados para profesionales dentro del programa, en los que pasan las tardes conversando sobre sus vidas cotidianas cada vez que acuden al CAP para una consulta de rutina. Incluso algunas veces escuché a una de las referentes de la organización llamar a Merlina "mami”.

18 Trabajo.

${ }^{19}$ La palabra "piola” en este caso remite a una cosa buena, que entusiasma.
} 
programa. Esos "lazos" con referentes de la "sociedad civil", llevaron a los profesionales a desarrollar nuevas modalidades de atención. Merlina lo recuerda entre lágrimas:

Aprendí mucho de mis pacientes. Me enseñaron a valorar derechos humanos. Me enseñaron a trabajar en equipo, a veces a no ser, a no tener el modelo médico hegemónico y eso nos enseñó mucho a nuestro equipo. A trabajar con los psicólogos, la trabajadora social, la enfermera, son fundamentales. (Entrevista Merlina 24/10/2016)

Estas nuevas relaciones transformaron así las prácticas cotidianas, incluido el trabajo en equipo, y los llevaron a reconsiderar las necesidades de sus pacientes, redefiniendo sus prioridades y orientaciones en la atención. Con el tiempo, estas relaciones pasarían a formar parte de aquello que llaman "redes de amistad y contactos". Nuevamente se destaca en los relatos de los profesionales la imposibilidad de establecer una distinción entre aquello que sería instrumental y aquello que pertenece al orden de los afectos. Estas relaciones son al mismo tiempo producto de los requerimientos técnicos de los proyectos y del afecto generado entre los profesionales y los pacientes organizados. Es en este entramado que se despliega la construcción local de la respuesta al VIH/Sida.

En cuanto a qué le "enseñaron" los "pacientes", Matilde alude: "a no tener el modelo médico hegemónico", expresión que puede entenderse como una crítica a un modo de accionar basado en el biologicismo - uno de los rasgos que Menéndez (MENÉNDEZ, 1990) atribuye a este modelo. Y señala antes aun en el extracto: "me enseñaron a valorar derechos humanos", una cuestión que marca el "compromiso" que a su juicio requiere la atención a la salud en el seno del Estado. Retomaremos esto en la conclusión.

\section{El proceso de atención: "te seguis manejando por redes de amistad, que es más efectivo y manoseás menos al paciente"}

Las "redes de amistad" constituyen un componente significativo en el curso de la tarea cotidiana, ya que - junto con otros mecanismos fundados en la autoridad de la que Merlina dispone dentro del municipio - permiten al programa sostener su forma de atención.

He señalado que los integrantes del Programa Municipal realizan tareas de gestión de gobierno de la epidemia (como la apertura de centros de testeo rápido en otros CAP, actividades de capacitación, gestión y provisión local de insumos como reactivos de laboratorio y preservativos); así como tareas asistenciales propias de un servicio de salud (atención médica, psicológica y orientación de demandas sociales). Es decir, cumplen funciones como programa gubernamental y como servicio de salud ${ }^{20}$. Este doble rol de asistencia-gestión está íntimamente relacionado con los orígenes del programa y el hecho de

\footnotetext{
${ }^{20}$ Esta práctica es frecuente en programas de escala provincial en el interior del país, pero no así en los programas de escala municipal de la provincia de Buenos Aires.
} 
que, por un lado, haya sido creado por un funcionario municipal - como programa sanitario - pero cuyo "germen", como señala Oscar, haya estado vinculado a la asistencia. Es también este doble carácter el que les permite tener contacto cotidiano y fluido con las personas que demandan atención y al mismo tiempo con funcionarios y agentes estatales de diferentes niveles burocráticos (municipales, regionales, provinciales y nacionales).

En lo referido a las tareas de asistencia, cabe señalar que los circuitos de atención estipulados por el Programa Municipal son diferentes a los del CAP, aunque coexisten en un mismo espacio. La atención general del CAP funciona por medio de la solicitud de turnos programados en la administración, cuenta con servicio social, área psicológica y farmacia propias. Existen requisitos que se solicitan para el acceso a servicios como el laboratorio y el área de radiología: que el domicilio de residencia (constatable en el Documento Nacional de Identidad) sea dentro del área del municipio y que la persona demuestre no tener ninguna otra cobertura de más que los servicios públicos de salud ${ }^{21}$. Por su parte, el Programa Municipal realiza atención por "demanda espontánea”, es decir, sin turnos programados. Esto incluye la asistencia médica, psicológica y el asesoramiento social, dispensa de medicación y distribución de recursos preventivos como preservativos y folletería. Esta práctica está sustentada en la idea que Merlina expresa diciendo:

Sé que muchos de mis pacientes venían de lugares muy pobres, de lugares muy alejados [...] entonces el día que conseguían su moneda para viajar, ese día podían venir. (Entrevista Merlina 24/10/2016)

La única condición formal que el programa coloca a las personas que acuden allí es que no posean otra cobertura de salud al momento de recibir la medicación antirretroviral, requisito que es estipulado por el Ministerio de Salud de la Nación para su provisión.

En este marco, los profesionales deben realizar distintas acciones cotidianas que permiten a "sus pacientes" sortear algunos de los requisitos que el CAP coloca para la atención y que a su juicio obstaculizarían su acceso a recursos como la vacunación antigripal, la leche en polvo y suplementos alimentarios y medicamentos. Simultáneamente, Merlina abastece al Programa con muestras farmacéuticas, que en la mayoría de los casos le permite satisfacer las necesidades de la población sin depender de la farmacia del CAP. Recuperando los aportes de Paiva y Ayres (AYRES, 2001; 2006, 2003; PAIVA et al., 2018; 2006), podríamos caracterizar el accionar de estos profesionales como una forma de respuesta integral a la vulnerabilidad diferencial de la población que acude al programa.

\footnotetext{
${ }^{21}$ El sistema de salud en Argentina es el resultado de la coexistencia de tres subsistemas: privado, de obras sociales y público. El primero consiste en empresas que presentan cobertura de salud a personas que realizan el pago voluntario de la misma. El segundo se compone por trabajadores en relación de dependencia, está financiado por las contribuciones de empleadores, trabajadores y del Estado. Finalmente, el sistema público está financiado con recursos provenientes del Estado y su cobertura es universal. Para información sobre la composición del Sistema de Salud en Argentina véase Ministerio de Salud de la Nación. 2010. Politicas de Salud: Programa Médicos Comunitarios. Módulo 5. Programa Médicos Comunitarios. Buenos Aires: Ministerio de Salud de la Nación.
} 
Lograr que "sus pacientes" sean eximidos de los requisitos señalados, implica para los profesionales desafiar algunos criterios y modalidades de atención del municipio y en particular del CAP, además de negociaciones constantes con todo tipo de actores: directores de programas, directores del centro de salud, administrativos y responsables de servicios de atención.

Durante los días en el programa, es frecuente que los profesionales echen mano de sus relaciones personales con trabajadores, funcionarios y profesionales de otros efectores de salud, municipios e instancias gubernamentales de todo tipo, para sostener el acceso pronto a ciertos recursos, a la atención o a la derivación de sus pacientes. Así lo observé en múltiples ocasiones, cuando un paciente llegaba al programa con alguna necesidad frecuente: vacunarse, recibir el tratamiento para sífilis, dar con un medicamento o una derivación a otro profesional. Matilde rápidamente me explicó que lo mejor para no exponer a las personas a un posible "maltrato, discriminación o estigmatización" era derivarlos con profesionales que conocieran personalmente, con quienes supieran que recibirían una atención adecuada. Las posibilidades de derivación incluyen, bajo el mismo criterio, asociaciones como Narcóticos Anónimos y organizaciones de PVIH.

Este tipo de decisiones son valoradas positivamente por Matilde, señalando la importancia de resolver ante todo los problemas de salud y las demandas de los pacientes, evitando incurrir en procedimientos burocrático-adminstrativos que podrían demorar ese proceso. En la entrevista lo expresa en los siguientes términos:

Te seguís manejando por redes de amistad, que es más efectivo y manoseás menos al paciente, y lo hacés ir menos, y donde lo van a atender realmente lo van a atender, y no le van a hacer mayor historia. (Entrevista Matilde $23 / 11 / 2016)$

Esto implica una acción concreta, que en palabras de Matilde se expresa como "levantar el teléfono y llamar" a quienes pueden "dar respuestas" inmediatas, salteando las burocracias y permitiendo con esa acción acortar los tiempos de espera o garantizar atención adecuada para las personas involucradas. Para poder obtener esas respuestas inmediatas, algunas veces se apela a la autoridad del programa y su larga trayectoria en el municipio o a la autoridad de Merlina como médica infectóloga municipal "desde hace más de 30 años". Esta legitimidad se sostiene en las mismas relaciones personales que dieron forma al programa a través de la historia; es inseparable de la "red de amistades" que los profesionales establecieron a comienzos de la epidemia y también de los "contactos" con autoridades y funcionarios municipales.

Por otra parte, se acude a relaciones de "amistad" y/o de afinidad profesional con profesionales de otros efectores de salud (municipales, provinciales o nacionales) o del propio CAP. También se apela a "conocidos" o "compañeros" a quienes Merlina brinda atención cuando le solicitan "ayuda" o le piden un "favor". En múltiples ocasiones observé 
cómo Merlina atendía o conseguía medicamentos para enfermeros, administrativos, autoridades y funcionarios locales. Camila comentaba en este sentido que "a Merlina la quieren todos, porque ella siempre atiende a todo el mundo", o señalaba que ciertas personas del CAP "tienen buena onda" con el programa gracias a la atención que Merlina les brinda. Es habitual que trabajadores administrativos, enfermeros y autoridades municipales acudan al programa a solicitar medicamentos, atención, o incluso llamen por teléfono para realizar consultas médicas. Ante estas situaciones Merlina generalmente brinda respuestas, aun cuando esto le genere molestias o le parezca una pérdida de tiempo ${ }^{22}$.

Por su parte, quienes reciben esa atención usualmente devuelven el gesto de un modo $\mathrm{u}$ otro: a veces con regalos para los profesionales, $\mathrm{u}$ ofreciendo resolver por ellos alguna gestión administrativa. En alguna oportunidad también escuché decir "cualquier cosa en lo que te pueda ayudar, me avisás" a modo de agradecimiento. Una de las posibilidades es que estos contrafavores puedan eventualmente ser canalizados por el programa como atención preferencial para los pacientes con VIH.

Los principios que regulan estas relaciones se construyen a partir de valoraciones sobre las formas apropiadas e inapropiadas de realizar favores y devolverlos (QUIRÓS, 2014) invistiéndolos de una dimensión no sólo instrumental o utilitaria sino también de sentimientos y valores. Así, en una ocasión una enfermera me dijo, luego de que Merlina la atendiera (y cuando ella no estaba presente): "ella es muy buena compañera”. Estas acciones que hemos llamado "favores" y "contrafavores" pueden entenderse como dones, asentados en bases morales que constituyen la ética de los agentes y que se ofrecen sin garantías de recibir algo a cambio (COMAS-D'ARGEMIR, 2017) pero revestidos por cierto sentido de obligatoriedad (MAUSS, 2007).

Consideramos que este conjunto de relaciones, en todas sus variantes, actúan como un puente entre el programa y el CAP y constituyen un componente significativo de los circuitos cotidianos de atención de las PVIH. Es significativo recordar en este sentido que se trata de un programa que se encuentra al mismo tiempo dentro y fuera del CAP, ya que comparte el espacio físico y brinda atención como otros servicios, al tiempo que desarrolla circuitos propios de atención y gestiona recursos estatales de distinto nivel.

El desarrollo de estos circuitos, organizados en torno de la premisa de brindar atención "buena y oportuna" a sus pacientes, implica una permanente articulación del universo de las relaciones personales con el entramado institucional. A través de este ejercicio los profesionales construyen las modalidades particulares de atención del programa y configuran - al mismo tiempo - las políticas municipales de respuesta al VIH.

\footnotetext{
${ }^{22}$ Observé una ocasión en la que Merlina se negó a realizar un "favor" cuando una compañera le solicitó que reiterara un certificado médico para justificar ausencias prolongadas en su puesto de trabajo. Merlina señaló el pedido como un exceso, no sin antes exponer la situación frente al equipo de trabajo del programa.
} 


\section{Palabras finales - "[nosotros] somos la cara del Estado"}

Hemos señalado, apoyándonos en una perspectiva etnográfica, las formas en que las relaciones personales modelan la respuesta al VIH en la localidad bajo estudio, y cómo mis interlocutores otorgan sentido a las mismas (BALBI, 2008b). Así, observamos cómo las relaciones personales pusieron en marcha el Programa Municipal; cómo estas mismas relaciones les habilitaron el desarrollo de proyectos financiados por entidades internacionales y cómo se movilizan las redes de "favores" y "relaciones" en la tarea cotidiana, dando respuesta a las necesidades de atención y a las condiciones de vulnerabilidad de sus pacientes. Esto permite ver el modo en que los actores producen las políticas locales articulando el mundo de las relaciones personales con el entramado institucional y nos permite acceder a una mejor comprensión de las respuestas políticas al VIH y a la vez a la densidad del Estado (FASSIN, 2015).

Al mismo tiempo, nos hemos acercado a la concepción que estos profesionales tienen sobre sí mismos y sobre el Estado. Al referirse a la elaboración y ejecución de proyectos financiados por entidades de cooperación global, Matilde señala que el programa es "contraparte del Estado" frente a las organizaciones internacionales y también de la sociedad civil - durante la misma entrevista dice, asimismo: "[nosotros] somos la cara del Estado, y creemos que tenemos que atender bien y brindar un buen servicio" (Entrevista Matilde 23/11/2016) ${ }^{23}$. Así, "somos el Estado" o "la cara del Estado" son la expresión de una identificación de sí, que dota de sentido y pone en valor la práctica institucional y el ejercicio profesional cotidianos. Enlazados con las adscripciones políticas, las historias personales y las trayectorias profesionales, estos sentidos se fundan en concepciones sobre la salud pública y la noción de salud como "derecho humano" que debe ser garantizado. Bajo este paraguas de historias, relaciones y adscripciones, una "buena atención” se traduce en las prácticas cotidianas como tener "compromiso", trabajar "a pulmón” o "por amor al arte”. Quien se reconoce como "profesional comprometido" se valoriza a sí por la entrega desinteresada y la defensa "militante" de aquello que constituye política y jurídicamente un derecho universal.

\section{Referencias bibliográficas}

ABRAMS, Philip. Notas sobre la dificultad de estudiar al estado. Virajes, Año 2, n. 2, 1977.

ADASZKO, Ariel. Trayectorias de vida y manejo de la enfermedad en la vida cotidiana de adolescentes que crecieron viviendo con VIH/sida. Actualizacionesen Sida, v.75, p.19-32, 2012.

ASAD, Talal. ¿Dónde están los márgenes del estado? Cuadernos de Antropología Social, n. 27, p. 53-62, 2008.

AUYERO, Javier. La politica de los pobres: las prácticas clientelistas del peronismo. [s.l.] Ediciones Manantial, 2001.

Clientelismo político en Argentina: doble vida y negación colectiva. Revista Perfiles Latinoamericanos, v. 10, n. 20, p. 33-52, 2002.

\footnotetext{
${ }^{23}$ El resaltado es nuestro.
} 
Patients of the State: an ethnographic account of poor people's waiting. Latin American Research Review, v. 46, n. 1, p. 5-29, 2011.

AYRES, José Ricardo. Sujeito, intersubjetividade e práticas de saúde. Ciência \& Saúde Coletiva, v. 6, n. 1, p. 63-72, 2001.

AYRES, José Ricardo, et al. Vulnerability human rights, and comprehensive health care needs of young people living with HIV/AIDS. American Journal of Public Health, v. 96, n. 6, p. 1001-1006, 2006.

AYRES, José Ricardo, et al. O risco, vulnerabilidade e práticas de prevenção e promoção da saúde. Saúde em debate, v. 170, p. 375-417, 2003.

BALBI, Fernando Alberto; Perspectivas en el análisis etnográfico de la producción social del carácter ilusorio del Estado. Revista de Estudios Marítimos y Sociales, v. 3, n. 3, p. 7-17, 2008.

BALBI, Fernando Alberto; BOIVIN, Maurucio. La perspectiva etnográfica en los estudios sobre política, Estado y gobierno. Cuadernos de Antropología Social, v. 27, p. 7-17, 2008b.

BARBER, Nélida. Experiencias de enfermedad y procesos de constitución de subjetividades. [s.l.] Universidad de Buenos Aires., 2015.

BARRÓN LOPEZ, Sara; LIBSON, Micaela; HILLER, Renata. Estudio social en hombres que tienen sexo con hombres (HSH) relevamiento 2007. Buenos Aires: UBATEC, 2008.

BIAGINI, Graciela. El hospital público y la significación social del VIH/sida. Cuadernos Médicos Sociales, n. 78, p. 55-71, 2000.

BIAGINI, Graciela. Sociedad civil y VIH-SIDA: de la acción colectiva a la fragmentación de intereses? Buenos Aires: Paidós, 2009.

BIAGINI, Graciela; GRAGAITIS, Laura; GIRI, Beatriz. Embarazo y VIH/sida, vivencias del proceso salud-enfermedad-atención: un estudio de casos de mujeres seropositivas embarazadas atendidas en efectores públicos seleccionados. Buenos Aires: UBATEC SA, 2008.

BIAGINI, Graciela.; SÁNCHEZ, Maria. Actores sociales y SIDA. Las organizacones no gubernamentales en Argentina y el complejo VIH-sida. Buenos Aires: Espacio Editorial, v. 23, p. 45, 1995.

BIEHL, João. Antropologia no campo da saúde global. Horizontes Antropológicos, v. 17, n. 35, p. 227-256, 2011.

CANELO, Brenda. Dirigentes de migrantes andinos, empleados y funcionarios públicos ante " el Estado ". Una mirada desde abajo para comprender procesos políticos locales (ciudad de Buenos Aires, Argentina). Cuadernos de Antropología Social, n. 27, p. 175193, 2008.

COMAS-D'ARGEMIR, Dolors. El don y la reciprocidad tienen género: Las bases morales de los cuidados. Quaderns de l'Institut Catala d'Antropologia, v. 22, n. 2, p. 17-32, 2017.

COUTO, Bárbara. Producción y ocupación en el conurbano bonaerense: radiografía de un territorio heterogéneo. Observatorio del Conurbano Bonaerense UNGS. [s.l: s.n.]. 
Disponível em: <http://observatorioconurbano.ungs.edu.ar/?p=8494>.

CROJETHOVIC, María. El aspecto informal de las organizaciones públicas: instrumento de dominación y estrategia para el cambio. Cuadernos del CENDES, v. 29, n. 79, p. 1-19, 2012.

EPELE, María. Cambios en las Prácticas de Uso de Cocaína: Neo-liberalismo , VIH-SIDA y Muerte en el Sur del Gran Buenos Aires. v. 38, n. 9, p. 1189-1216, 2003.

EPELE, M. La logica de la sospecha. Sobre criminalización y barreras de acceso al sistema de salud. Cuadernos de Antropología Social, n. 25, p. 151-168, 2007.

FASSIN, Didier. When Bodies Remember: Experiences and Politics of AIDS in South Africa. Berkley: University of California Press, 2007.

. At the Heart of the State: the moral world of institutions. London: Pluto Press, 2015.

FUNDACIÓN HUÉSPED. [Stigma index among people living with HIV in Argentina]. 2011.

FUNDACIÓN HUÉSPED. Conocimientos y actitudes sobre las hepatitis A , B y C en población general y personal de salud. Fundación Husped, 2014.

FUNDACIÓN HUÉSPED. Análisis de la accesibilidad y la calidad de atención de la salud para la población lesbiana, gay, trans y bisexual (LGBT) en cinco regiones sanitarias de la Provincia de Buenos Aires. 2017.

FUNDACIÓN HUÉSPED; UNICEF. Conocimientos. Actitudes y prácticas en VIH y Salud Sexual y Reproductiva (SSR) y uso de Tecnologías de la Información y la Comunicación (TIC) entre Adolescentes de Argentina. [s.l: s.n.].

GARCÍA, Guadalupe. Cuerpo y narrativa: una aproximación etnográfica al proceso de atención del embarazo, parto y puerperio de mujeres viviendo con vih en la ciudad de Buenos Aires. Horizontes Antropológicos, v. 15, n. 32, p. 247-272, 2009.

De políticas globales e itinerarios de atención: etnografía de la prevención de la transmisión perinatal del VIH en un hospital público de Buenos Aires. Revista Colombiana de Antropología, v. 53, p. 63-84, 2017.

; RECODER, María Laura; MARGULIES, Susana. Espacio, tiempo y poder en la atención hospitalaria de la salud y la enfermedad: Aportes de una etnografía de un centro obstétrico. Salud Colectiva, v. 13, n. 3, p. 391, 2017.

GAZTAÑAGA, Julieta ¿ Qué es el trabajo político ? Notas etnográficas acerca de militantes y profesionales de la política. Cuadernos de Antropología Social, n. 27, p. 133-153, 2008.

GOGNA, Mónica (Org). Embarazo y maternidad en la adolescencia: estereotipos, evidencias y propuestas para políticas públicas. Buenos Aires: CEDES Buenos Aires, 2005.

; PANTELIDES, Alejandra.; RAMOS, S. Las enfermedades de transmisión sexual: género, salud y sexualidad. Buenos Aires: CENEP,1997.

GREGORIC, Juan José. Entre " la parte social " y " lo que tiene que ver con políticas ". Una aproximación a las acciones y demandas de personas que viven con VIH-SIDA de sectores. Anales de IX Congreso Argentino de Antropología Social. Posadas: Universidad Nacional de Misiones, 2008. 
Biosociabilidad y demandas por derechos en el activismo de personas de sectores populares afectadas por VIH-SIDA. Cuadernos de Antropología Social, n. 35, p. 121-142, 2012.

Vih-Sida y activismo: Un estudio antropológico del proceso de organización y la politización de la salud entre personas viviendo con el Vih. [s.l.] Universidad de Buenos Aires. Facultad de Filosofía y Letras, 2015.

Activismo de personas afectadas por el VIH. [s.l.] Universidad de Buenos Aires, 2017.

GRIMBERG, Mabel. Sexualidad y construcción social del HIV-SIDA: las representaciones médicas. Cuadernos médicos sociales, n. 70, p. 37-51, 1995.

Género y vih/sida: un análisis de los diferenciales de género en la experiencia de vivir con vih. Cuadernos médicos sociales, n. 78, p. 41-54, 2000.

. «Saber de SIDA»y cuidado sexual en mujeres jóvenes de sectores populares del cordón sur de la ciudad de Buenos Aires. Apuntes para la definición de políticas de prevención. Cadernos de Saúde Pública, v. 17, p. 481-489, 2001.

Iniciación sexual, prácticas sexuales y prevención al VIH/SIDA en jóvenes de sectores populares: un análisis antropológico de género. Horizontes antropológicos, v. 8 , n. 17 , p. 47-75, 2002a.

. VIH-Sida, vida cotidiana y experiencia subjetiva. Una revisión conceptual de las dimensiones de vivir con VIH. Cuadernos Médico Sociales, v. 82, p. 43-59, 2002b.

Sexualidad, experiencias corporales y género: un estudio etnográfico entre personas viviendo con VIH en el Área Metropolitana de Buenos Aires, Argentina. Cadernos de Saúde Pública, v. 25, p. 133-141, 2009.

GRIMBERG, M.; MARGULIES, S. S. Género, sexualidad y Vih-Sida. Un análisis antropológico de las representaciones y prácticas de mujeres y varones de sectores populares. Buenos Aires: [s.n.].

GRIMBERG, Mabel.; WALLACE, S.; MARGULIES, S. S. Construcción social y hegemonía. Representaciones médicas sobre el SIDA. Un abordaje antropológico. En: KORNBLIT, Ana Lia. (Ed.). Sida y Sociedad. Buenos Aires: Ed. Espacios/ Instituto de Investigaciones de la Facultad de Ciencias Sociales, 1997.

GUPTA, Akhil. Fronteras borrosas el discurso de la corrupción, la cultura de la política y el Estado imaginado. En: ABRAMS, Philip.; GUPTA, Akhil.; MITCHELL, Timothy. (Eds.). Antropología del Estado. México: Fondo de cultura Económica, 2015. p. 71-143.

JONES, Daniel. Adherencia a los tratamientos antirretrovirales en personas que viven con VIH en la Argentina. Buenos Aires: Ministerio de Salud de la Nación, Dirección de Sida y ETS, 2017.

JONES, Daniel; BARRÓN LOPEZ, Sara.; IBARLUCÍA, Inés. Significados asociados al VIH y al sida en la Argentina Significados asociados al VIH y al sida en la Argentina: resumen ejecutivo. Ciudad Autónoma de Buenos Aires: Ministerio de Salud de la Nación, Dirección de Sida y ETS, 2016. 
¿Qué piensan y hacen las personas ante el VIH y el sida?: un estudio sobre significados asociados al VIH y al SIDA en población general en la Argentina. Ciudad Autónoma de Buenos Aires: Ministerio de Salud de la Nación, Dirección de Sida y ETS, 2017.

JORRAT, Jorge Raul. et al. Información, actitudes y conductas en relación con el VIH-sida: Estudio social en población bajo la línea de pobreza en el área metropolitana de Buenos Aires. Ciudad Autónoma de Buenos Aires: UBATEC SA, 2013. v. 84

KORNBLIT, Ana Lia. Sida y Sociedad. Buenos Aires: Ed. Espacios/ Instituto de Investigaciones de la Facultad de Ciencias Sociales, 1997.

KORNBLIT, Ana Lia.; GIMÉNEZ, Liliana. Y el sida está entre nosotros: un estudio sobre actitudes, creencias y conductas de grupos golpeados por la enfermedad. [s.l.] Corregidor, 1997.

KORNBLIT, Ana Lia; MENDES-DIZ, A.; PETRACCI, M. La gestión del riesgo frente al VIH. En: KORNBLIT, Ana Lia (Coord). Sida: entre el cuidado y el riesgo -estudios en población general y en personas afectadas. Buenos Aires: Alianza Editorial, 2000. p. 97 110.

KORNBLIT, Ana Lia; PETRACCI, Mónica. Las ONGs que trabajan en el campo del VIH/SIDA: una tipología. IV Simposio Nacional de Análisis Organizacional, II del Cono Sur, Buenos Aires: Facultad de Ciencias Económicas, UBA. Anais...1999

LAPLACETTE, G. et al. Respuesta del Estado al VIH-Sida, ITS y hepatitis virales en la Argentina. [s.l.] Ministerio de Salud de la Nación, Dirección de Sida y ETS, 2015.

LOWY, Ilana. Trustworthy knowledge and desperate patients: clinical tests for new drugs from cancer to AIDS. En: LOCK, M.; YOUNG, A.; CAMBROSIO, A. (Eds.). Living and working with the new medical technologies: Intersections of Inquiry. Cambridge ed. Cambridge: [s.n.]. p. 49-81.

MANZANO, Virginia. La politica en movimiento: movilizaciones colectivas y políticas estatales en la vida del Gran Buenos Aires. 1era. ed. Rosario: Prohistoria Ediciones, 2013.

MARGULIES, Susana. La adherencia a los tratamientos: un desafío para la atención del VIH/Sida . Una lectura desde la antropología. p. 63-69, 2010.

. La construcción médica del Vih-Sida. Buenos Aires: Mimeo, 1996.

. Candidaturas y VIH-SIDA tensiones en los procesos de atención. Cuadernos Médico Sociales, v. 063, p. 1-10, 1998.

La atención del VIH-SIDA. un estudio de antropología de la medicina. Ciudad Autónoma de Buenos Aires: Editorial de la Facultad de Filosofa y Letras Universidad de Buenos Aires, 2014.

Etiología y riesgo en la construcción clinica de la enfermedad VIH-sida: Ensayo de antropología de la medicina. 2017.

MARGULIES, Susana. et al. Sexualidad y vulnerabilidad al vih en personas homosexuales/gay, bisexuales y trans. Ciudad Autónoma de Buenos Aires: Ministerio de Salud de la Nación, Dirección de Sida y ETS, 2010. v. I

MARGULIES, Susana; BARBER, Nélida; RECODER, Maria Laura.VIH/Sida y “adherencia” 
al tratamiento - Enfoques y perspectivas. Antipoda, v. 3, n. 3, p. 281-300, 2006.

MARGULIES, Susana; STIVAL, Matias.; NAME, Maria Júlia. El acceso a la atención de la salud en personas homosexuales/gay, bisexuales y trans: una aproximación desde los equipos de salud. Ciudad Autónoma de Buenos Aires: Ministerio de Salud de la Nación, Dirección de Sida y ETS, 2010. v. II

MARQUÉS SÁNCHEZ, P. et al. Las relaciones informales: un valor añadido en la gestión de personas. Enfermeria Global, v. 11, n. 2, p. 310-323, 2012.

MAUSS, Marcel. Intoduccion: Sobre el don y, en particular, sobre la obligación de devolver los regalos. Ensayo sobre el don: Forma y función del intercambio en las sociedades arcaicas, p. 67-79, 2007.

MENDES-DIZ, A. M.; CAMAROTTI, A. C.; SCHW ARZ, P. Los usuarios de drogas y el riesgo de transmisión del VIH/sida. Ciudad Autónoma de Buenos Aires: UBATEC SA, 2008.

MENÉNDEZ, Eduardo. Morir de Alcohol. Saber y Hegemonía Médica. DF México: Consejo Nacional para la Cultura y las Artes, 1990.

MITCHELL, T. Sociedad Economía y el efecto del estado. En: ABRAMS, P.; GUPTA, A.; MITCHELL, T. (Eds.). Antropología del Estado. México: Fondo de cultura Económica, 2015. p. 17-18.

NGUYEN, Vinh-kim. Antiretroviral globalism, biopolitics and therapeutic citizenship. En: ONG, Aihwa; COLLIER, Stephen. (Eds.). Global Assemblages: Technology, Politics, and Ethics as Anthropoloragical Problems. Malden: Blackwell, p. 124-144. 2005.

PAIVA, Vera. et al. Prevención, promoción y cuidado: enfoques de vulnerabilidad y derechos humanos. Temperley: TesseoPress, 2018.

PAIVA, Vera; PUPO, Lígia; BARBOZA, Renato. The right to prevention and the challenges of reducing vulnerability to HIV in Brazil. Rev Saude Publica, v. 40 Suppl, n. 0034-8910 (Print), p. 109-119, 2006.

PANTELIDES, Edith.; GOGNA, Mónica; RAMOS, Silvania. Concepciones legas de salud y enfermedad: el SIDA según pobladores de un barrio pobre del Gran Buenos Aires. Reproducción, salud y sexualidad en América Latina, p. 117-135, 2000.

PECHENY, Mario et al. Caracterización de las mujeres recientemente diagnosticadas con VIH en ArgentinaResumen ejecutivo. [s.l: s.n.].

PECHENY, Mario.; JONES, Daniel.; MANZELLI, Hernan. Vida cotidiana con VIH-Sida y/o hepatitis C: diagnóstico, tratamiento y proceso de expertización. Seminario (5: 2002Buenos Aires, AR). Anais...Centro de Estudios de Estado y Sociedad, 2002

QUIRÓS, Julieta. Piqueteros y peronistas en la lucha del Gran Buenos Aires . Por una visión no instrumental de la política popular. Cuadernos de Antropología Social, n. 27, p. 113131, 2008.

El porqué de los que van. Peronistas y piqueteros en el Gran Buenos Aires (una antropología de la política vivida). Buenos Aires: Antropofagia, 2014.

RECODER, Maria Laura. El problema médico de la adherencia,'contrato terapéutico'y participación en la atención a personas que viven con VIH-SIDA. Cuadernos de 
Antropología Social, v. 13, 2001.

Vivir con VIH-sida Notas etnográficas sobre el mundo de la enfermedad y sus cuidados. [s.l: s.n.].

ROVERE, Mario. Redes en salud: Un nuevo paradigma para el abordaje de las organizaciones y la comunidad. Rosario: Secretaría de Salud Pública/Instituto Lazarte, 1999.

SANCHEZ, M. Políticas de salud y sociedad civil: El caso del VIH-sida. p. 0-20, 2013.

SHORE, C. La antropología y el estudio de las políticas públicas: reflexiones sobre la «formulación» de las políticas públicas. Antipoda: Revista de Antropologia y Arqueologia, v. 10, n. 10, p. 21-49, 2010.

SPINELLI, H. Gestión: Prácticas, mitos e ideologías. Salud Colectiva, v. 13, n. 4, p. 577-597, 2017.

SULLIVAN, N. Enacting Spaces of Inequality: Placing Global / State Governance Within a Tanzanian Hospital. 2012.

VALLE, Carlos Guilherme. Identidades, doença e organização social: um estudo das «Pessoas Vivendo com HIV e AIDS». Horizontes Antropológicos, v. 8, p. 179-210, 2002.

Biosocial Activism, Identities and Citizenship: Making up «people living with HIV and AIDS» in Brazil. Vibrant: Virtual Brazilian Anthropology, v. 12, n. 2, p. 27-70, 2015.

VOMMARO, G.; QUIRÓS, J. “Usted vino por su propia decisión”: repensar el clientelismo en clave etnográfica. Desacatos, v. 36, p. 65-84, 2011.

WELLER, S. et al. ¿Dónde estamos? ¿Adónde queremos ir? Respuesta al VIH/sida desde el sistema público de salud. Buenos Aires: UBATEC SA, 2009.

autora

\section{Agostina Aixa Gagliolo}

Es doctoranda del Programa de Antropología de la Salud, sección de Antropología Social del Instituto de Ciencias Antropológicas de la Universidad de Buenos Aíres 